\title{
Trimethoprim-Sulfamethoxazole (TMP-SMX) Induced Severe Systemic
} Reaction

\author{
Farrukh Abbas ${ }^{1 *}$, Mehul Patel ${ }^{1}$, Khuram Abbas $^{2}$, Pulin Shah ${ }^{1}$ and Michael N Gurel
}

${ }^{1}$ Rochester General Hospital, Rochester, NY, USA

${ }^{2}$ Shalimar Hospital, Lahore, Pakistan

\begin{abstract}
Objective: Trimethoprim-Sulfamethoxazole (TMP-SMX) is a relatively commonly prescribed antibiotic. One of the rare but dramatic reactions to TMP-SMX is severe systemic reaction which can mimic sepsis or septic shock and this can be a diagnostic challenge especially in critical care setting. The objective is to raise information about this side effect.

Study Selection: The case series includes two patients' reports. The first patient was 85 year old woman who received TMP-SMX before dental extraction and presented with rash and fever. She met SIRS criteria with leukocytosis, bandemia, lactic acidosis and acute kidney injury. All the infectious work remained negative. She improved with supportive care. She received TMP-SMX again after a few weeks and again presented with similar clinical course. Again the infectious work up remained negative and she improved with supportive care. It was thought that TMP-SMX contributed to this.

The second patient was 85 year old man who took TMP-SMX for right leg cellulitis and presented with systemic inflammatory response resembling septic shock. All the infectious work up remained negative. The patient recovered completely with supportive care. TMP-SMX was thought to be responsible for the side effect.

Conclusion: TMP-SMX induced systemic reaction resembling anaphylaxis or sepsis is rare. Symptoms are very similar to classic sepsis and septic shock including fever, chills, leukocytosis and hemodynamic instability. This has been described mostly in HIV patients but this may happen in non-HIV patients as well.
\end{abstract}

Keywords: Bactrim; Trimethoprim-sulfamethoxazole; SIRS; Sepsis like syndrome; Septic shock; Drug reaction

\section{Background}

Trimethoprim-Sulfamethoxazole (TMP-SMX) is a relatively commonly prescribed antibiotic. TMP-SMX related adverse effects are more common in HIV infected patients than non-HIV infected patients. One of the rare but dramatic reaction to TMP-SMX can resemble anaphylaxis or sepsis and has been well described in patients infected with human immunodeficiency virus (HIV) [1,2]. We report such unusual reaction to TMP-SMX in two patients who were not infected with HIV.

\section{Case 1}

An 85 year old woman with history of bio-prosthetic mitral valve presented with fever and rigors four hours after she took trimethoprimsulfamethoxazole (TMP-SMX) before dental work up. On physical examination, she had maculopapular rash on her face and left arm. Her labs showed leukocytosis of $22,300 / \mathrm{ul}$ with $23 \%$ bands, lactic acid of 5.2 $\mathrm{mmol} / \mathrm{L}$, Creatinine of $1.3 \mathrm{mg} / \mathrm{dl}$ with baseline creatinine of $0.9 \mathrm{mg} /$ $\mathrm{dl}$ and potassium of $4.3 \mathrm{mEq} / \mathrm{L}$. She was initially managed with broad spectrum antibiotics which were discontinued after 48 hours. Blood cultures and urine cultures remained negative for any bacterial growth. CT abdomen, CT chest and transthoracic echocardiography failed to show any source of infection. She was managed with supportive care with no recurrence of symptoms, resolution of leukocytosis and normalization of creatinine. She was discharged home after four days with the diagnosis of severe sepsis with unclear source.

About 4 weeks later, the patient again presented with rigors five hours after taking TMP-SMX again for her dental work. Physical examination was remarkable for facial erythema. Her labs showed leukocytosis of $18,000 / \mathrm{ul}$ with $85 \%$ neutrophils, $13 \%$ bands, lactic acid of $2.8 \mathrm{mmol} / \mathrm{L}$, Creatinine of $1.2 \mathrm{mg} / \mathrm{dl}$ and potassium of $4.1 \mathrm{mEq} / \mathrm{L}$. Blood cultures, urine culture and chest imaging failed to show any evidence of infection. She was given ciprofloxacin for one day for possible sepsis which was then discontinued as there was no source of infection including normal urinalysis and no growth on urine culture. Liver function tests were normal. Her symptoms and laboratory abnormalities resolved with supportive care and she was discharged home after 24 hours. Infectious disease was consulted during both admissions and her presentation was thought to be due to TMP-SMX induced systemic reaction resembling sepsis.

\section{Case 2}

An 85 year old man with no significant past medical history was admitted to the emergency department with symptoms of altered mental status, lethargy and hypotension. On admission, he had leukocytosis and elevated lactate. Liver function tests were unremarkable. Notably, he was discharged the day prior, for an admission of right thigh cellulitis, which was treated with intravenous Vancomycin prior to being discharged home on oral Bactrim. The patient did not have sepsis from cellulitis on initial presentation and leg cellulitis seemed to be improving before discharge. Patient had taken single dose of Bactrim before presenting with above symptoms. In addition, patient developed erythroderma like reaction with fluid filled blisters in skin on his trunk and arms (Figure 1). Patient required aggressive intravenous fluid administration and vasopressor support. Infectious work up including

*Corresponding author: Farrukh Abbas, Rochester General Hospital, Rochester NY, USA, Tel: 585-957-3160; E-mail: farrukh.abbas@rochesterregional.org

Received December 27, 2016; Accepted February 08, 2017; Published February 11, 2017

Citation: Abbas F, Patel M, Abbas K, Shah P, Gurel MN (2017) TrimethoprimSulfamethoxazole (TMP-SMX) Induced Severe Systemic Reaction. J Pulm Respir Med 6: 393. doi: 10.4172/2161-105X.1000393

Copyright: $\odot 2017$ Abbas F, et al. This is an open-access article distributed under the terms of the Creative Commons Attribution License, which permits unrestricted use, distribution, and reproduction in any medium, provided the original author and source are credited. 
Citation: Abbas F, Patel M, Abbas K, Shah P, Gurel MN (2017) Trimethoprim-Sulfamethoxazole (TMP-SMX) Induced Severe Systemic Reaction. J Pulm Respir Med 6: 393. doi: 10.4172/2161-105X.1000393

Page 2 of 2

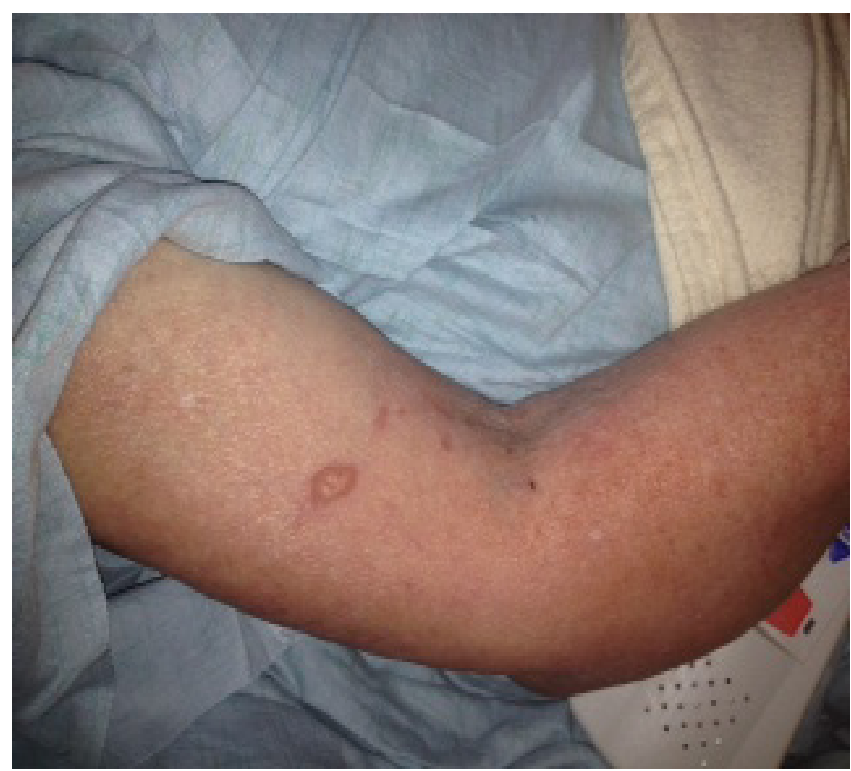

Figure 1: Erythroderma like reaction with fluid filled blisters.

chest X-ray, urinalysis and blood culture data remained negative. The cellulitis of the leg had improved since presentation and it was thought that it did not contribute towards current presentation.

He was hemodynamically stable 72 hours after re-admission and discharged without any antibiotics. It was thought that the patient's presentation was likely from TMP-SMX.

\section{Discussion}

Adverse reactions to Trimethoprim-Sulfamethoxazole (TMPSMX) are more common in HIV infected patients than non-HIV infected patients and most common reactions include maculopapular rash with or without fever. TMP-SMX induced severe systemic reaction resembling anaphylaxis or sepsis has been described in HIV infected patients $[1,2]$. Symptoms are very similar to classic sepsis and septic shock including fever, chills, leukocytosis, bandemia and hemodynamic instability. Based on literature search, almost all of patients improved within 72 hours after discontinuation of TMP-SMX. There is no clear

\begin{tabular}{|c|c|c|}
\hline Characteristics & Anaphylaxis & Sepsis Like syndrome \\
\hline Eosinophilia & Raised & Normal \\
\hline IgE levels & Raised & Normal \\
\hline Temperature & Often normal & Hyperthermia \\
\hline Blood pressure & Hypotension & Hypotension \\
\hline Heart rate & Tachycardia & Tachycardia \\
\hline Laryngeal edema & Common & Uncommon \\
\hline Bronchospasm & Common & Uncommon \\
\hline Urticaria & Common & Uncommon \\
\hline Erythema & Common & Common \\
\hline
\end{tabular}

Table 1: Difference between anaphylaxis and sepsis like syndrome.

explanation of this severe systemic reaction. In HIV infected patients, it is thought to be due to hypersensitivity reaction resulting from autoxidation of the hydroxylamine metabolite of sulfamethoxazole [3]. There is no clear explanation as to why this reaction happened in nonHIV positive population.

In our patient described in first, well established sepsis like syndrome occurred with initial and recurrent exposure to TMPSMX. We do not believe our patients' illness represented true sepsis. It not unclear why the patient was prescribed TMP-SMX before dental procedure because this not a commonly prescribed prophylactic agent and moreover, this patient did not need antibiotic prophylaxis for the prevention of endocarditis according to American Heart Association guidelines. Anaphylactic reactions are common with use of Bactrim. Table 1 helps us distinguish sepsis like syndrome from the more common anaphylactic reaction to TMP-SMX.

This severe systemic reaction to TMP-SMX can be a diagnostic challenge in critical care settings.

\section{References}

1. Kelly JW, Dooley DP, Lattuada CP (1992) A severe unusual reaction to trimethoprim-sulfamethoxazole in patients infected with human immunodeficiency virus. Clin Infect Dis 14: 1034-1039.

2. Cohn DL, Penley KA, Judson FN, Kirkpatrick $\mathrm{CH}$, Horsburgh $\mathrm{CR}$, et al (1984) The acquired immunodeficiency syndrome and a trimethoprimsulfamethoxazole adverse reaction. Ann Intern Med 100: 311.

3. Lehmann DF, Liu A, Newman N, Blair DC (1999) The association of opportunistic infections with the occurrence of trimethoprim/sulfamethoxazole hypersensitivity in patients infected with human immunodeficiency virus. J Clin Pharrmacol 39: 533-537. 